Canad. Math. Bull. Vol. 19 (4), 1976

\title{
TENSOR PRODUCTS AND BIMORPHISMS
}

\author{
BY \\ BERNHARD BANASCHEWSKI AND EVELYN NELSON
}

Introduction. The binary tensor product, for modules over a commutative ring, has two different aspects: its connection with universal bilinear maps and its adjointness to the internal hom-functor. Furthermore, in the special situation of finite-dimensional vector spaces, the tensor product can also be described in terms of dual spaces and the internal hom-functor. The aim of this paper is to investigate these relationships in the setting of arbitrary concrete categories.

A tensor multiplication for a functor $H: \mathbf{K}^{*} \times \mathbf{K} \rightarrow \mathbf{K}\left(\mathbf{K}^{*}\right.$ the opposite of the category $\mathbf{K}$ ) is a functor $T: \mathbf{K} \times \mathbf{K} \rightarrow \mathbf{K}$, essentially unique if it exists, for which there is a natural equivalence

$$
\mathbf{K}(T(A, B), C) \rightarrow \mathbf{K}(A, H(B, C)) .
$$

On the other hand, for any concrete category $\mathbf{K}$ with underlying set functor $\mid$, a bimorphism is a (set!) map $f:|A| \times|B| \rightarrow|C|$ for objects $A, B, C$ in $\mathbf{K}$ such that, for each $a \in|A|$,

$$
f(a,)=\left|u_{a}\right| \text { for some } u_{a}: B \rightarrow C,
$$

and, analogously,

$$
f(, b)=\left|v_{b}\right| \text { for some } v_{b}: A \rightarrow C,
$$

for each $b \in|B|$. Evidently, this notion gives rise to a set-valued functor BiM on $\mathbf{K}^{*} \times \mathbf{K}^{*} \times \mathbf{K}: \operatorname{BiM}(A, B, C)$ is the set of all bimorphisms $f:|A| \times|B| \rightarrow|C|$, and the effect on maps $u: A^{\prime} \rightarrow A, v: B^{\prime} \rightarrow B$, and $w: C \rightarrow C^{\prime}$ is $f \leadsto|w| f(|u| \times|v|)$.

A bimorphism $f:|A| \times|B| \rightarrow|C|$ is called universal (for $A$ and $B$ ) iff any bimorphism $g:|A| \times|B| \rightarrow|D|$ has a factorization $g=|h| f$ with a unique $h: C \rightarrow D$. If universal bimorphisms exist for each pair of objects in $\mathbf{K}$, they determine a functor $U: \mathbf{K} \times \mathbf{K} \rightarrow \mathbf{K}$ together with a natural transformation $\beta:|| \times|| \rightarrow|U()$,$| such that the components of \beta$ are the universal bimorphisms, and the map $U(f, g): U(A, B) \rightarrow U(C, D)$ is given by the factorization $\beta_{C D}(|f| \times|g|)=|U(f, g)| \beta_{A B}$, for any $f: A \rightarrow C$ and $g: B \rightarrow D$. Moreover, the correspondence $h \leadsto|h| \beta_{A B}$ is then a natural equivalence $\mathbf{K}(U(A, B), C) \rightarrow$ $\operatorname{BiM}(A, B, C)$, and the universal bimorphisms are entirely determined by this, $\beta_{A B}$ being the map corresponding to the identity map of $C=U(A, B)$. In the

Received by the editors July 17, 1975 and, in revised form, May 3, 1976. 
following, $U$ will be called the functor of universal bimorphisms, and $\beta_{A B}$ will always be the associated universal bimorphism for $A$ and $B$.

Of course, there is no connection a priori between tensor multiplication and universal bimorphisms, but in certain situations they turn out to be equivalent notions, in the sense that the functor $U$ is the tensor multiplication for $H$. Apart from the case referred to above, this holds for a varietal category iff the homomorphisms $A \rightarrow B$ always form a subobject of $B^{|A|}$ (Linton [8]). Hence the general question: For an arbitrary concrete category $\mathbf{K}$, how should a functor $H: \mathbf{K}^{*} \times \mathbf{K} \rightarrow \mathbf{K}$ be related to the underlying set functor in order that tensor products and universal bimorphisms be equivalent? A natural requirement is that $H$ should be an internal hom-functor, i.e. $|H()|=,\mathbf{K}($, ), but this is not sufficient, as is shown by any concrete category with universal bimorphisms which has several different such $H$ with tensor multiplication. Examples are the category of mono-binary relational structures (Pultr [13]), and the category of Hausdorff spaces with the pointwise and the compact-open function space topologies (Brown [3]).

In this paper, we consider a concrete category $\mathbf{K}$ with internal hom-functor $H$. First, we discuss certain conditions for $H$ which make the objects $H(A, B)$ resemble pointwise structure on sets of functions (Section 1) and show these are sufficient, and in some sense necessary, for the equivalence of tensor products and universal bimorphisms (Proposition 3). Second, we show that an object $D$ in $\mathbf{K}$ for which the cofunctor ()$^{*}=H(, D)$ is a selfduality of $\mathbf{K}$ determines a tensor multiplication $T$ of $H$, given by $T(A, B)=H\left(A, B^{*}\right)^{*}$ (Proposition 5). In addition, we show the existence of universal bimorphisms under fairly general conditions (Proposition 4) and present a number of examples (Section 4).

In the following, $A, B, \ldots$ will be objects and $f, g, \ldots$ maps of the category $\mathbf{K}$, and we let $(A, B)=\mathbf{K}(A, B)$, the set of all $f: A \rightarrow B$ in $\mathbf{K}$. General categorical notions will be used as in Mac Lane [9].

Thanks are due to the referee who alerted us to Linton [8] and otherwise made a number of helpful suggestions.

Financial support of the National Research Council of Canada is gratefully acknowledged.

1. Functional internal hom-functors. An internal hom-functor $H$ of a concrete category $\mathbf{K}$ (with underlying set functor | |) will be called functional iff it satisfies the following conditions:

(F1) For any $A, B \in \mathbf{K}$ there exist maps $e_{A B}: A \rightarrow H(H(A, B), B)$ such that ||$e_{\mathrm{AB}}|(a)|(u)=|u|(a)$ for all $a \in|A|$ and $u: A \rightarrow B$.

(F2) For any $A, B, C \in \mathbf{K}$, a set map $f:|A| \rightarrow|H(B, C)|$ is $|h|$ for some $h: A \rightarrow H(B, C)$ whenever, for each $b \in|B|, \quad|f()|(b)=\left|h_{b}\right|$ for some $h_{b}: A \rightarrow C$. 
If $\mathbf{K}$ has products which are concrete in the sense that the underlying set functor preserves them then these conditions essentially mean that the $H(A, B)$ have the usual pointwise structure on sets of functions. Indeed, call a monomorphism $f: A \rightarrow B$ an embedding iff any $g: C \rightarrow B$ factors through $f$ whenever $|g|$ factors through $|f|$. Then, with the present hypothesis on $\mathbf{K}$, one has the following characterization:

Proposition 1. An internal hom-functor $H$ of $\mathbf{K}$ is functional iff, for any $A$ and $B$ in $\mathbf{K}$, there is an embedding

$$
i_{A B}: H(A, B) \rightarrow B^{|A|}
$$

such that $\left|p_{a} i_{\mathrm{AB}}\right|(h)=|h|(a)$, for each $a \in|A|$ and $h: A \rightarrow B$, where $p_{a}$ is the projection for $a$.

Proof. $(\Rightarrow)(\mathrm{F} 1)$ directly implies the existence of these maps $i_{A B}$, and since $\left|p_{a} i_{A B} u\right|(c)=|| u|(c)|(a)$ for any $u: C \rightarrow H(A, B)$, they clearly are monomorphisms. To see that $i_{A B}$ is an embedding, consider any $g: C \rightarrow B^{|A|}$ such that $|g|=\left|i_{A B}\right| f, f:|C| \rightarrow|H(A, B)|$. From

$$
|f(c)|(a)=\left|p_{a} i_{A B}\right|(f(c))=\left|p_{a} g\right|(c) \quad(c \in|C|)
$$

it then follows that (F2) applies, hence $f=|h|$ for some $h: C \rightarrow H(A, B)$, and therefore $g=i_{A B} h$.

$(\Leftarrow)$ We first derive (F2). Given $f:|C| \rightarrow|H(A, B)|$ as in the hypothesis of that condition, one has the map $g: C \rightarrow B^{|A|}$ such that $p_{a} g=h_{a}$ where $|f()|(a)=\left|h_{a}\right|$, and simple computation shows that $\left|p_{a} g\right|=\left|p_{a} i_{A B}\right| f$; since || preserves products it then follows that $|g|=\left|i_{A B}\right| f$ and hence $f=|h|$ for some $h: C \rightarrow H(A, B)$. To obtain (F1), note that the map $v:|A| \rightarrow(H(A, B), B)$, $v(a)=p_{a} i_{A B}(a \in|A|)$, satisfies the hypothesis of (F2), which then provides the desired $e_{\mathrm{AB}}: A \rightarrow H(H(A, B), B)$ since $|v(a)|(h)=|h|(a)$.

REMARK. The last part of the above proof shows that, in the presence of (F2), (F1) follows from the apparently weaker condition: for any $A, B \in K$ and $a \in|A|$ there exists a map $\tilde{a}: H(A, B) \rightarrow B$ such that $|\tilde{a}|(h)=|h|(a)$. On the other hand, if the category $\mathbf{K}$ is as above and has the additional property that all monomorphisms are embeddings then (F1) evidently implies (F2).

We note that many naturally arising internal hom-functors are functional, as the discussion in the last section will show; on the other hand, examples to the contrary also occur readily, e.g. the internal hom-functor of the category of topological spaces given by the compact-open topology.

Next, we turn to another characterization of functionality of internal homfunctors, now for an arbitrary concrete category $\mathbf{K}$, which demonstrates the significance of the functor BiM. 
Proposition 2. An internal hom-functor $H$ is functional iff there is a natural equivalence $\quad(A, H(B, C)) \rightarrow \operatorname{BiM}(A, B, C)$ such that $h \rightsquigarrow \underline{h}, \underline{h}(a, b)=$ ||$h|(a)|(b)$, for all $a \in|A|$ and $b \in|B|$.

Proof. $(\Rightarrow)$ For any $h: A \rightarrow H(B, C)$ the set map $h$ is indeed a bimorphism since $\underline{h}(a)=,|| h|(a)|$ and $\underline{h}(, b)=|| e_{\mathrm{BC}}|(b) h|$. Also, the correspondence $h \leadsto \underline{h}$ is evidently one-one, and its naturality is easily checked. Hence it only remains to show $h \leadsto h$ is onto. If $g:|A| \times|B| \rightarrow|C|$ is any bimorphism, define $f:|A| \rightarrow|H(B, C)|$ such that $f(a)=u_{a}$ where $\left|u_{a}\right|=g(a$,$) . Then, the second$ variable part of the bimorphism condition for $g$ shows that $(\mathrm{F} 2)$ is applicable to $f$, and thus $f=|h|$ for some $h: A \rightarrow H(B, C)$. Simple calculation now proves that $g=\underline{h}$.

$(\Leftarrow)$ We first show that $(\mathrm{F} 2)$ holds. If $f:|A| \rightarrow|H(B, C)|$ satisfies the hypothesis in question then $\bar{f}:|A| \times|B| \rightarrow|C|, \bar{f}(a, b)=|f(a)|(b)$, is clearly a bimorphism, and hence $\bar{f}=\underline{h}$ for some $h: A \rightarrow H(B, C)$ by the current assumption. This implies that $f=|h|$. To obtain (F1), let $A=H(B, C)$ and $h$ be the identity map; then it follows, from the fact that $\underline{h}$ is a bimorphism, that there exists a map $v_{b}: H(B, C) \rightarrow C$ such that $\left|v_{b}\right|(u)=|u|(b)$, for each $b \in|B|$. By the remark after Proposition 1, this is sufficient to obtain (F1).

Basic facts about natural equivalences now imply the

COROLlary 1. Functional internal hom-functors, as far as they exist, are essentially unique.

The obvious natural equivalence $\operatorname{BiM}(A, B, C) \rightarrow \operatorname{BiM}(B, A, C)$, given by "interchange of variables", has the following consequence:

CoRollary 2. For any functional internal hom-functor $H$, there is a natural isomorphism $H(A, H(B, C)) \rightarrow H(B, H(A, C))$ with underlying set map $f \leadsto f^{\#}$, ||$f^{\#}|(b)|(a)=|| f|(a)|(b)$ for all $a \in|A|$ and $b \in|B|$.

Proof. The stated set map is the composite $\phi$ of the three natural equivalences

$$
(A, H(B, C)) \rightarrow \operatorname{BiM}(A, B, C) \rightarrow \operatorname{BiM}(B, A, C) \rightarrow(B, H(A, C)),
$$

and its inverse is of the same form. Hence, we only have to exhibit a map $H(A, H(B, C)) \rightarrow H(B, H(A, C))$ with underlying set map $\phi$. For this, note that

$$
|\phi(f)|(b)=\tilde{b} f=|H(1, \tilde{b})|(f)
$$

for any $f: A \rightarrow H(B, C)$ and $b \in|B|$, so that $|\phi()|(b)=|H(1, \tilde{b})|$ for any $b \in|B|$, and hence the desired map exists by (F2).

REMARK. The existence of the above isomorphism will be called the exponent law for $H$. That this holds for functional internal hom-functors in varietal categories is contained in Linton [8]; moreover, in that situation, the exponent 
law implies, conversely, the functionality of an internal hom-functor [8]. We do not know whether the same holds here, but note that, from the exponent law, one obtains the map $e_{B C}$ of (F1) for $A=H(B, C)$, considering the identity map of $H(B, C)$. Thus, by the Remark following Proposition 1 , if $\mathbf{K}$ has concrete products, and all its monomorphisms are embeddings, then the exponent law implies $H$ is functional. Since varietal categories satisfy these conditions, this covers the case of the converse of Corollary 2 given in [8].

2. Universal bimorphisms. We first establish the precise interdependence between universal bimorphisms, tensor products, and functionality for any concrete category $\mathbf{K}$ with internal hom-functor $H$.

Proposition 3. For an internal hom-functor $H$ of $\mathbf{K}$, and any functor $S: \mathbf{K} \times$ $\mathbf{K} \rightarrow \mathbf{K}$, any two of the following conditions imply the third:

(T) $S$ is a tensor multiplication for $H$

(F) $H$ is functional

(U) $S$ is the functor of universal bimorphisms.

Proof. This results immediately from the explicit description of these conditions in terms of natural isomorphisms:

(T) $(S(A, B), C) \rightarrow(A, H(B, C))$

(F) $(A, H(B, C)) \rightarrow \operatorname{BiM}(A, B, C)$

(U) $\operatorname{BiM}(A, B, C) \rightarrow(S,(A, B), C)$.

REMARK 1. If (F) and (U) hold then (T) is explicitly given by $h \leadsto \tilde{h}$ where ||$\tilde{h}|(a)|(b)=|h| \beta_{\mathrm{AB}}(a, b), \beta$ the natural transformation giving the universal bimorphisms. Similarly, if $(\mathrm{F})$ and $(\mathrm{T})$ hold then the universal bimorphism on $|A| \times|B|$ is $(a, b) \leadsto|| \eta_{A B}|(a)|(b)$ where $\eta_{A B}: A \rightarrow H(B, S(A, B))$ corresponds to the identity map of $C=S(A, B)$ through (U) and $(\mathrm{F})$, respectively. Incidentally, the map $h \leadsto \tilde{h}$ in (T) actually lifts to $\mathbf{K}:|\tilde{h}|(a)=\left|h u_{a}\right|$ for the map $u_{a}$ such that $\left|u_{a}\right|=\beta_{A B}(a$,$) , and hence (F2) applies. The resulting map will be an$ isomorphism whenever $\mathbf{K}$ has the property that the isomorphisms are exactly the one-one onto maps, but in general there seems to be no way to construct an inverse.

Remark 2. Pumplün [14] proves the equivalence of (T) and (U) assuming somewhat different conditions on $H$, i.e. (1) the set maps $(A, B) \rightarrow$ $(H(B, C), H(A, C))$ and $(A, B) \rightarrow(H(C, A), H(C, B))$, by $f \leadsto H(f, C)$ and $f \leadsto$ $H(C, f)$ respectively, underly maps in $\mathbf{K}$, and (2) for some $K \in \mathbf{K}, H(K, \quad)$ is equivalent to the identity functor. Here, the functionality of $H$ implies (1) (see Lemma 1 for the first part, the second being analogous) so that [14] implies the result in question, for functional $H$ satisfying (2). 
The following immediate consequence of Proposition 3 and previous remarks is an abstract version of the Theorem in Linton [8], as far as it can be translated into the present setting.

COROLlaRY. If the category $\mathbf{K}$, with universal bimorphism functor $U$ and internal hom-functor $H$, has concrete products and all its monomorphisms are embeddings then the following are equivalent:

(1) $H$ is functional.

(2) $U$ is a tensor multiplication for $H$.

(3) H satisfies the exponent law.

We conclude this section with a result on the existence of universal bimorphisms in a concrete category $\mathbf{K}$. There are various different ways of ensuring this existence but we consider only one of them in detail.

First we recall the following notions, essentially due to Herrlich [6]: A (possibly proper) class $\mathbf{M}$ of maps $f: A \rightarrow C$ with common domain is called a monosource iff $f g=f h$ for all $f \in \mathbf{M}$ implies $g=h . \mathbf{K}$ is said to have epimonosource factorization iff, for any class $\mathbf{L}$ of maps $f: A \rightarrow C$ with common domain, there exists an epimorphism $h: A \rightarrow B$ such that each $f \in \mathbf{L}$ factors through $h$, and the class of all $\bar{f}: B \rightarrow C, f \in \mathbf{L}$ and $f=\bar{f} h$, is a monosource.

Proposition 4. If $\mathbf{K}$ has coproducts and epi-monosource factorization, and its monosources are preserved by the underlying set functor, then $\mathbf{K}$ has universal bimorphisms.

Proof. To begin with, we note that a bimorphism $|A| \times|B| \rightarrow|C|$ can be viewed as a pair of families of maps in $K, u_{a}: B \rightarrow C(a \in|A|)$ and $v_{b}: A \rightarrow C$ $(b \in|B|)$, subject to the condition that $\left|u_{a}\right|(b)=\left|v_{b}\right|(a)$ for all $a \in|A|$ and $b \in|B|$.

Now, for any $A$ and $B$ in $\mathbf{K}$, let $E$ be the coproduct of the family, indexed by the disjoint union of $|A|$ and $|B|$, defined by $E_{(a, 0)}=B$ for all $a \in|A|$, and $E_{(b, 1)}=A$ for all $b \in|B|$, with the coproduct maps $i_{a}: B \rightarrow E$ and $j_{b}: A \rightarrow E$. Then, let $f=\bar{f} h, h: E \rightarrow F$ and $\bar{f}: F \rightarrow C$ be an epi-monosource factorization of the class of all $f: E \rightarrow C$ such that $\left|f i_{a}\right|(b)=\left|f j_{b}\right|(a)$ for all $a \in|A|$ and $b \in|B|$. Since || preserves monosources one then also has $\left|h i_{a}\right|(b)=\left|h j_{b}\right|(a)$ for all $a \in|A|$ and $b \in|B|$, i.e. $h i_{a}(a \in|A|)$ and $h j_{b}(b \in|B|)$ constitute a bimorphism, and this is universal by the fact that any bimorphism $|A| \times|B| \rightarrow|C|$ determines a map $f: E \rightarrow C$ of the above kind.

Remark 1. There are various types of general conditions under which the above hypothesis concerning monosources holds fairly obviously; for instance, this is the case if $\mathbf{K}$ is cowellpowered, has concrete products and epi-mono factorization, and the underlying set tunctor preserves monomorphisms. However, usually that hypothesis is very easily checked directly. 
REMARK 2. The above construction of universal bimorphisms genuinely differs from the one ordinarily used in the case of modules over a commutative ring, where the existence of free modules, i.e. of a left adjoint to the underlying set functor, is employed. In the last section we give a simple example in which our construction applies but where the left adjoint fails to exist.

3. Dualizers. We now turn to a very particular situation in which a tensor multiplication arises. Again, we are dealing with a concrete category $\mathbf{K}$ with underlying set functor $\mid$ and functional internal hom-functor $H$.

For any object $D \in \mathbf{K}$, one has the cofunctor $H(, D)$ of $\mathbf{K}$ into itself, which is selfadjoint on the right by Corollary 2 of Proposition 2 and has the adjunction $e_{A D}: A \rightarrow H(H(A, D), D)$ given by (F1). $D$ will be called a dualizer iff this is a natural equivalence, i.e. iff all $e_{A D}$ are isomorphisms. In this case, the cofunctor $H(, D)$ is a dual equivalence of $\mathbf{K}$ with itself.

We consider a fixed $D$ with this property, and let $A^{*}=H(A, D), e_{\mathrm{A}}=$ $e_{A D}: A \rightarrow A^{* *}$, and $h^{*}=H\left(h, 1_{D}\right)$ for $h: A \rightarrow B$. The selfduality ()$^{*}=H(, D)$ of $\mathbf{K}$ is compatible with $H$, as described in

Lemma 1. For any $A, B \in \mathbf{K}$, there is an isomorphism $H(A, B) \rightarrow H\left(B^{*}, A^{*}\right)$ with underlying set map $h \rightsquigarrow h^{*}$.

Proof. For the given map $\phi$ one has $|\phi(h)|(u)=u h=|H(A, u)|(h)$ and hence (F2) applies. The resulting map has the composite of the analogous map $H\left(B^{*}, A^{*}\right) \rightarrow H\left(A^{* *}, B^{* *}\right)$ with the isomorphism $H\left(e_{A}, e_{B}^{-1}\right)$ as inverse and hence is an isomorphism.

REMARK. The natural isomorphisms $H\left(D^{*}, A\right) \cong H\left(A^{*}, D^{* *}\right) \cong H\left(A^{*}, D\right) \cong$ $A^{* *} \cong A$ show that $D^{*}$ represents the identity functor via $H$, and that $\left(D^{*},\right)$ is naturally equivalent to the underlying set functor; the latter makes $D^{*}$ essentially unique. The map $\left(D^{*}, A\right) \rightarrow|A|$, incidentally, is $h \leadsto|h|\left(1_{D}\right)$, as simple calculation shows. Conversely, if one has a self-duality $S$ of $\mathbf{K}$, compati- . ble with the given internal hom-functor $H$ in the sense of Lemma 1, and a representation $H(I, \quad)$ of the identity functor, then $D=S I$ is a dualizer of $\mathbf{K}$ and $S \cong H(, D)$.

A further result concerning the cofunctor ()$^{*}$ is

LemmA 2. For any $A \in \mathbf{K}$, the map $e_{A^{*}}^{*} e_{\mathrm{A}^{*}}: A^{*} \rightarrow A^{*}$ is the identity.

Proof. For any $\phi \in\left|A^{*}\right|$ and any $a \in|A|$, one has

$$
\begin{aligned}
\| e_{\mathrm{A}}^{*} e_{\mathrm{A}^{*}}|(\phi)|(a) & =|| e_{\mathrm{A}^{*}}\left|(\phi) e_{\mathrm{A}}\right|(a) \\
& =|| e_{\mathrm{A}^{*}}|(\phi)|\left(\left|e_{\mathrm{A}}\right|(a)\right) \\
& =|| e_{\mathrm{A}}|(a)|(\phi)=|\phi|(a),
\end{aligned}
$$

and therefore $\left|e_{A}^{*} e_{A^{*}}\right|(\phi)=\phi$, which proves the assertion. 
Our main interest here is in

Proposition 5. There is a natural isomorphism $H\left(H\left(A, B^{*}\right)^{*}, C\right) \rightarrow$ $H(A, H(B, C))$ with underlying set map $f \rightsquigarrow H\left(1_{B}, f\right) \eta_{A B}$ where $\eta_{A B}: A \rightarrow$ $H\left(B, H\left(A, B^{*}\right)^{*}\right)$ has the underlying set map $a \rightsquigarrow \hat{a},|\hat{a}|(b)=\left|e_{B}\right|(b)\left|e_{A B^{*}}\right|(a)$.

Proof. The existence of the natural isomorphism in question results from the following sequence of such isomorphisms based on Corollary 2 of Proposition 2, Lemma 1, and the natural isomorphism $e_{E}: E \rightarrow E^{* *}$ :

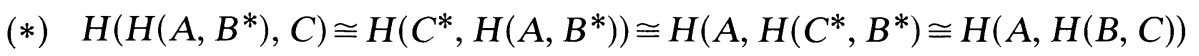

Concerning the specific form of the resulting isomorphism note that, for fixed $B$, the condition implies the left adjointness of the functor $H\left(, B^{*}\right)^{*}$ to the functor $H(B$,$) . The underlying set map is then of the type stated, with \eta_{A B}$ the associated front adjunction. In order to determine the latter, it remains to trace the identity map of $H^{*}=H\left(A, B^{*}\right)^{*}$ through the steps in $\left(^{*}\right)$, for the case $C=H^{*}$. This produces the sequence

$$
1_{H^{*}} \rightsquigarrow e_{H}^{-1} 1_{h^{*}}^{*}=e_{H}^{-1} \rightsquigarrow\left(e_{H}^{-1}\right)^{\#} \leadsto j_{B H^{*}}\left(e_{H}^{-1}\right)^{\#}=\eta_{A B}
$$

where \# is as in Corollary 2 of Proposition 2 and $j_{B H^{*}}$ is the isomorphism $H\left(H^{* *}, B^{*}\right) \rightarrow H\left(B, H^{*}\right)$ resulting from Lemma 1, i.e. $\left|j_{B H^{*}}^{-1}\right|(u)=u^{*}$ for $u \in$ $\left(B, H^{*}\right)$. The latter implies, by the proof of Lemma 1 , that $\left|j_{B H^{*}}\right|(s)=e_{H^{*}}^{-1} e_{B}$.

Now for any $a \in|A|$ and $v \in H^{* *}$,

$$
||\left(e_{H}^{-1}\right)^{\#}|(a)|(v)=|| e_{H}^{-1}|(v)|(a)=|| e_{\mathrm{AB}}|(a)|\left(e_{H}^{-1} \mid(v)\right)=|| e_{\mathrm{AB}} *\left|(a) e_{H}^{-1}\right|(v),
$$

so that $\left|\left(e_{H}^{-1}\right)^{\#}\right|(a)=\left|e_{\mathrm{AB}}\right|(a) e_{H}^{-1}$. It follows that, for any $b \in|B|$,

$$
\begin{aligned}
\| j_{\mathrm{B} H^{*}}\left(e_{H}^{-1}\right)^{\#}|(a)|(b) & =\| j_{\mathrm{BH}^{*}}\left|\left(\left|\left(e_{H}^{-1}\right)^{\#}\right|(a)\right)\right|(b)=\left|e_{H^{*}}^{-1}\left(\left|e_{\mathrm{AB}}\right|(a) e_{H}^{-1}\right)^{*} e_{\mathrm{B}}\right|(b) \\
& =\left|e_{H^{*}}^{-1}\left(e_{H}^{-1}\right)^{*}\right| e_{\mathrm{AB}^{*}}\left|(a)^{*} e_{B}\right|(b)=\left|e_{B}\right|(b)\left|e_{\mathrm{AB}}\right|(a),
\end{aligned}
$$

the last step as a consequence of Lemma 2. This shows that ||$\eta_{A B}|(a)|(b)=$ $\left|e_{\mathrm{B}}\right|(b)\left|e_{\mathrm{AB}}\right|(a)$.

By Proposition 3, we now have the following immediate consequence:

COROllary. The cofunctor ( )* provides universal bimorphisms $\beta_{A B}:|A| \times$ $|B| \rightarrow\left|H\left(A, B^{*}\right)^{*}\right|$ where $\beta_{A B}(a, b)=\left|e_{B}\right|(b)\left|e_{A B}\right|(a)$.

4. Examples and Remarks. In this section, we discuss a number of situations which illustrate our results.

(1) If $\Omega$ is a (possibly proper) class and $\tau=\left(n_{\lambda}\right)_{\lambda \in \Omega}$ any $\Omega$-indexed family of cardinal numbers then the category $\operatorname{Alg}(\tau)$ of algebras of type $\tau$ has as its objects the algebras $A=\left(X,\left(\xi_{\lambda}\right)_{\lambda \in \Omega}\right)$ with underlying set $|A|=X$ and $\lambda$ th 
operation $\lambda_{A}=\xi_{\lambda}: X^{n_{\lambda}} \rightarrow X$, for each $\lambda \in \Omega$, its maps being the homomorphisms $h: A \rightarrow B$, which are, up to labelling to indicate domain and codomain, the set maps $|h|:|A| \rightarrow|B|$ such that all diagrams

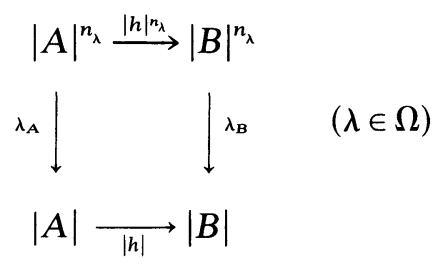

commute. In the present context, a category of algebras is always meant to be a full, isomorphism-closed subcategory $\mathbf{K}$ of a category $\operatorname{Alg}(\tau)$ which is productive and hereditary, i.e. closed with respect to arbitrary (cartesian) products and subalgebras. If $\mathbf{K}$ has the property that for each cardinal number $k$ there exists a cardinal number $m(k)$ such that any $A \in \mathbf{K}$ generated by a set of at most $k$ elements has itself at most $m(k)$ elements then the underlying set functor | | of $\mathbf{K}$ has a left adjoint, and conversely, as is clear from the Adjoint Functor Theorem. Such $\mathbf{K}$ will be called bounded; evidently, if $\Omega$ is only a set then all $\mathbf{K} \subseteq \operatorname{Alg}(\tau)$ are bounded. If $\mathbf{K}$ is bounded and, in addition, closed in $\operatorname{Alg}(\tau)$ with respect to homomorphic images then $\mathbf{K}$ is a varietal category, the case considered in Linton [8].

A category $\mathbf{K}$ of algebras evidently has the property that all monomorphisms are embeddings. Hence, by Proposition $1, \mathbf{K}$ has a functional internal homfunctor $H$ iff for any family $\left(h_{i}\right)_{i \in n_{\lambda}}$ of homomorphisms $h_{i}: A \rightarrow B(A, B \in \mathbf{K}$ and $\lambda \in \Omega)$ the composite map

$$
|A| \stackrel{h}{\longrightarrow}|B|^{n_{\curlywedge}} \stackrel{\lambda_{B}}{\longrightarrow}|B|
$$

( $h$ the map whose composite with the $i$ th projection is $h_{i}$ ) is again a homomorphism, since this is precisely the condition which makes the image of $(A, B)$ in $B^{|A|}$ a subalgebra $H(A, B)$ of the latter. By considering what this means for the projections $|B|^{n_{\mu}} \rightarrow|B|(\mu \in \Omega)$ one obtains the following criterion, due to Linton [8] for the varietal case: $\mathbf{K}$ has a functional internal hom-functor iff the identity

$$
\lambda_{B}\left(\mu_{B}\left(x_{i j}\right)_{j \in n_{\mu}}\right)_{i \in n_{\lambda}}=\mu_{B}\left(\lambda_{B}\left(x_{i j}\right)_{i \in n_{\lambda}}\right)_{j \in n_{\mu}}
$$

holds for all $x_{i j} \in|B|$ and all $\lambda, \mu \in \Omega$. This shows there is a largest category of algebras, for each type $\tau$, with a functional internal hom-functor, namely the subcategory of $\operatorname{Alg}(\tau)$ (equationally!) defined by the above "interchange laws".

Any category $\mathbf{K}$ of algebras satisfies the hypothesis of Proposition 4 concerning monosources. The required factorization of a class $\mathbf{L}$ of homomorphisms $f: A \rightarrow C$ with common domain is obtained by first taking the quotient algebra 
of $A$ modulo the congruence consisting of the $(x, y)$ such that $f(x)=f(y)$ for all $f \in \mathbf{L}$, and then reflecting into $\mathbf{K}$. The preservation of monosources by the underlying set functor results from the fact that the triviality of the above congruence characterizes monosources: if $f(x)=f(y)$ for all $f$ but $x \neq y$ then the subalgebra $B$ of $A \times A$ generated by $(x, y)$ has two different homomorphisms into $A$ which are equalized by all $f$. Thus, by Proposition $4, \mathbf{K}$ has universal bimorphisms whenever it has coproducts, and this is certainly the case for any bounded $\mathbf{K}$. An unbounded example is as follows. Let $R$ be the (large!) commutative polynomial ring $\mathbb{Z}[X]$ in a proper class of indeterminates $X$, i.e. the union of the updirected system of polynomial rings $\mathbb{Z}[Y]$ where $Y$ ranges over the subsets of $X$. Then the category $R$ Mod of (small) $R$-modules is a category of algebras with functional internal hom-functor. $R$ Mod has coproducts, given by direct sums as for ordinary module categories, but it is unbounded-there are arbitrarily large singly generated $R$-modules.

We conclude this part with the following negative fact concerning dualizers in categories of algebras: If all operations are finitary, and epimorphisms of $\mathbf{K}$ are onto maps, then $\mathbf{K}$ does not have a dualizer. This is a consequence of a result of Fajtlowicz [4], but we present an independent proof for it. Take any non-trivial $A \in \mathbf{K}$, and let $F$ be the free algebra functor to the productive, hereditary subcategory of $\mathbf{K}$ generated by $A$. For any infinite set $X$, consider the projective limit $P=\lim _{\rightarrow} F(X / R)$ in $K$, with limit maps $p_{R}: P \rightarrow F(X / R)$, where $X / R$ ranges over the finite quotient sets of $X$, with the natural maps $X / Q \rightarrow X / R$ for $Q \subseteq R$. Then the embeddings $X / R \rightarrow|F(X / R)|$ lift to an embedding $\beta X \rightarrow P$, since projective limits are constructed on the underlying sets and $\beta X=\lim _{\rightarrow} X / R$, for the set $\beta X$ of ultrafilters on $X$. It follows that card $P \geq \operatorname{card} \beta X>\operatorname{card} X$. On the other hand, from the homomorphisms $h_{R}: F X \rightarrow F(X / R)$, determined by the quotient maps $X \rightarrow X / R$, one has the homomorphism $h: F X \rightarrow P$ such that $h_{R}=p_{R} h$, for all $R$, Now, if $\mathbf{K}$ is self-dual then $h$ is an epimorphism, by duality, the properties of direct limits in $\mathbf{K}$ resulting from the finitariness of the operations, and the fact that all $p_{R} h=h_{R}$ are onto. However, since the operations on $A$ are already determined by a subset of $\Omega, X$ can be chosen such that card $F X=$ card $X$, and then $h$ cannot be onto.

(2) The category TOP of topological spaces and continuous maps has a functional internal hom-functor $H$ relative to the usual underlying set functor, the topology on the function space $H(X, Y)$ being the topology of simple (= pointwise) convergence, i.e. the restriction of the topology of the product space $Y^{|X|}$ to the set of the continuous maps, functionality resulting from Proposition 1.

TOP also has universal bimorphisms, by Proposition 4, and hence a tensor multiplication $T=U$ relative to $H$, by Proposition 3. This gives us the familiar closed structure of TOP. Explicitly, the construction in the proof of Proposition 
4 leads to the description of $T(X, Y)$ as a certain quotient space; however, it can also be seen directly that $|\mathrm{T}(\mathrm{X}, \mathrm{Y})|=|\mathrm{X}| \times|\mathrm{Y}|$ and the topology is the initial topology for the maps $x \rightsquigarrow(x, y)$ and $y \rightsquigarrow(x, y)(x \in|X|, y \in|Y|)$.

It might be added that TOP does not have a dualizer $D$. By the remark after Lemma $3, D^{*}$ would have to be a one-point space, but then so would $D$ itself, which is clearly impossible.

(3) Let CJSL be the category of complete join-semilattices whose objects are the complete partially ordered sets and whose maps are those $f: A \rightarrow B$ which preserve arbitrary joins, i.e. $f\left(\bigvee x_{\alpha}\right)=\bigvee f\left(x_{\alpha}\right)$ for any family $\left(x_{\alpha}\right)$ in $A$. Evidently, the complete partially ordered sets can be viewed as algebras in the sense of (1); for each cardinal $k$ there is one operation of arity $k$, namely $\left(x_{i}\right)_{i \in k} \rightsquigarrow \bigvee x_{i}$. Moreover, the maps of CJSL are then exactly the homomorphisms. Considered in this way, CJSL is in fact a category of algebras as defined in (1). Since joins in products of partially ordered sets are taken componentwise, $\prod A_{\alpha}$ as partially ordered set is also the product algebra of the $A_{\alpha} \in \mathrm{CJSL}$ viewed as algebras; likewise, any subalgebra of an $A \in$ CJSL is complete as partially ordered set and thus belongs to CJSL. Incidentally, it can be seen that this category is also closed relative to homomorphic images; this can be checked directly, but is also a consequence of the fact that the interchange laws, together with the idempotency laws $\bigvee x_{i}=x$ whenever all $x_{i}=x$ form a class of defining equations. Thus we have a characterization: CJSL is the largest category of algebras with functional internal hom-functor which has one idempotent operation of arity $k$ for each cardinal number $k$.

Concerning the use of Proposition 4, the discussion in (1) applies: CJSL is bounded-indeed, it is monadic (Mac Lane [9, p. 138], Manes [10])—and hence it has universal bimorphisms. It follows then, by Proposition 3, that the latter provide a tensor multiplication for its internal hom-functor. However, CJSL also has a dualizer, namely the two-element chain 2 , which then establishes the same facts, in reverse order, by Section 3. That 2 does have the asserted property is seen as follows. Any $a \in|A|$, for $A \in$ CJSL, determines a map $a^{*}: A \rightarrow 2$, defined such that

$$
a^{*}(x)=\left\{\begin{array}{lll}
0 & \text { for } & x \leq a \\
1 & \text { for } & x \neq \leq a
\end{array}\right.
$$

and $a^{*} \leq b^{*}$ in $H(A, 2)$ iff $b \leq a$. In fact, any $h: A \rightarrow 2$ is of this type, namely $h=a^{*}$ where $a=\bigvee x(h(x)=0)$, and hence the map $a \rightsquigarrow a^{*}$ is a dual isomorphism of partially ordered sets. Of course, this is not an isomorphism in CJSL, but its iterate $a \rightsquigarrow\left(a^{*}\right)^{*}$ is, and the desired result then follows by proving that this map is the same as the map $e_{A}: A \rightarrow A^{* *}$ given by $a \leadsto \tilde{a}$. One has, for any $a$ and $x$ in $|A|$,

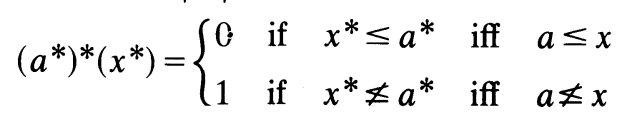


on the one hand, and $\tilde{a}\left(x^{*}\right)=x^{*}(a)$ on the other, which establishes the point.

The fact that CJSL has universal bimorphisms, and their relation to $H\left(A, B^{*}\right)^{*}$, was established by Mowatt [12]; the existence of the tensor multiplication in CJSL, derived from the dualizer 2 , is originally due to Waterman [18]. Without the consideration of the dualizer, these results also follow from Linton [8].

The category CJSL has a natural extension, namely the category POLA of bounded ordered sets and left adjoint (= residuated) maps, i.e. order preserving maps $f: A \rightarrow B$ such that $f(x) \leq y$ iff $x \leq f^{\#}(y)$ for a (necessarily unique) order preserving map $f^{\#}: B \rightarrow A$. The internal hom-functor of CJSL does not extend to POLA, although one can obviously talk about the partially ordered set $H(A, B)$ of all left adjoint $A \rightarrow B$, extending the object part of $H$ on CJSL. For $B=\mathbf{2}$ this is still functorial in $A$, and $\mathbf{2}$ remains a dualizer in this extended setting, so that any partially ordered sets $A$ and $B$ determine the partially ordered set $H\left(A, B^{*}\right)^{*}$; moreover, there is a natural map $|A| \times|B| \rightarrow$ $\left|H\left(A, B^{*}\right)^{*}\right|$, given by $(a, b) \leadsto \tau_{a b}, \tau_{a b}(h)=h(a)(b)$, which is a bimorphism for all $A, B \in$ POLA. The rôle of this is obscure, but we do know that it need not be a universal bimorphism, even if $A$ and $B$ are complete. In fact there is no universal bimorphism on $\mathbf{3} \times \mathbf{3}$ ( $\mathbf{3}$ the three-element chain). Some of these matters are discussed in Shmuely [17], albeit presented in rather different language.

(4) Let Ban be the category of all Banach spaces (over $K=\mathbb{R}$ or $\mathbb{C}$ ) and bounded linear maps. For any Banach spaces $A$ and $B$, one then has the Banach space $H(A, B)$ of all $f: A \rightarrow B$ in Ban, with the usual vector space structure and operator norm

$$
\begin{aligned}
\|f\| & =\inf \{\lambda \mid\|f(x)\| \leq \lambda\|x\| \quad \text { for all } x \in A\} \\
& =\sup \{\|f(x)\| \mid\|x\|=1\} .
\end{aligned}
$$

Moreover, $B$ an is concrete, with the obvious underlying set functor, and $(A, B) \leadsto H(A, B)$ is the object part of an internal hom-functor $H$ whose effect on maps is given by $H(f, g)(u)=$ guf for $u: A \rightarrow B, f: C \rightarrow A$ and $g: B \rightarrow D$, so that $\|H(f, g)\| \leq\|f\|\|g\|$. Furthermore, $H$ is functional.To obtain (F2), consider any $f:|A| \rightarrow|H(B, C)|$ such that $|f()(b)|=\left|h_{b}\right|, h_{b}: A \rightarrow C$, for each $b \in|B|$. $\|f(a)(b)\| \leq\left\|h_{b}\right\|\|a\|$ for any $a \in|A|$ and $b \in|B|$, and hence $\sup \{\|f(a)(b)\| \mid\|a\|=1\}$ is finite for each $b \in|B|$, which implies that $\sup \{f(a)\|\mid\| a \|=1\}$ is finite, by the Uniform Boundedness Principle (Dunford-Schwartz [3, Ch. II]). Thus, $f$ is bounded, and since it is obviously linear this proves (F2). For (F1), we now only have to note that, for any Banach spaces $A$ and $B$, evaluation at each $a \in A$ is a map $\tilde{a}: H(A, B) \rightarrow B$, and $a \leadsto \tilde{a}$ is a map, since $\mid \tilde{a}(h)\|=\| h(a) \| \leq$ $\|h\|\|a\|$.

Concerning the existence of universal bimorphisms, Proposition 3 cannot be 
applied here since Ban, although it has finite coproducts, fails to have coproducts for any infinite family. However, the proof of Proposition 3 can nonetheless be adapted to the present setting. We define a Banach space $E$ and maps $j_{b}: A \rightarrow E$ and $i_{a}: B \rightarrow E$ as follows: if $U$ and $V$ are the underlying vector spaces of $A$ and $B$ respectively, let $W$ be the vector space constructed from $U$ and $V$ as in the proof of Proposition 3, i.e. $W=\oplus W_{(a, 0)} \oplus W_{(b, 1)}$ where $W_{(a, 0)}=V$ and $W_{(b, 1)}=U$ for all $a \in|U|$ and $b \in|V|$, with the coproduct maps $k_{a}: V \rightarrow W$ and $\ell_{b}: U \rightarrow W$. Then define a norm on $W$ by additively extending the definition $\left\|k_{a}(b)\right\|=\|a\|\|b\|=\left\|\ell_{b}(a)\right\|$, and let $E$ be the corresponding completion of $W$; since the $k_{a}$ and $\ell_{b}$ are obviously bounded they now determine maps $i_{a}: B \rightarrow E$ and $j_{b}: A \rightarrow E$ in Ban.

From here we can proceed exactly as in the proof of Proposition 3 to obtain the map $h: E \rightarrow F$ since Ban clearly satisfies the required conditions regarding monosources. That the resulting bimorphism, given by the $h i_{a}$ and $h j_{b}$, is in fact universal requires some further consideration since $E$ is not a coproduct. Let $f:|A| \times|B| \rightarrow|C|$ be any bimorphism such that $f(a, b)=\left|u_{a}\right|(b)=\left|v_{b}\right|(a)$; again, by the Uniform Boundedness Principle, $\sup \left\{\left\|u_{a}\right\| \mid\|a\|=1\right\}$ is finite, i.e.

$$
\|f\|=\sup _{\|a\|=1} \sup _{\|b\|=1}\|f(a, b)\|
$$

is finite. It follows that the linear map $g$ determined on $W$ by $f$, i.e.

$$
x=k_{a_{1}}\left(b_{1}\right)+\cdots+\ell_{b_{k}}\left(a_{k}\right) \leadsto f\left(a_{1}, b_{1}\right)+\cdots+f\left(a_{k}, b_{k}\right),
$$

is bounded since

$$
\begin{aligned}
\|g(x)\| & \leq\left\|f\left(a_{1}, b_{1}\right)\right\|+\cdots+\left\|f\left(a_{k}, b_{k}\right)\right\| \\
& \leq\|f\|\left(\left\|a_{1}\right\|\left\|b_{1}\right\|+\cdots+\left\|a_{k}\right\|\left\|b_{k}\right\|\right) \\
& =\|f\|\|x\|,
\end{aligned}
$$

and therefore extends to a map $E \rightarrow C$ in Ban. This is enough to show that the bimorphism determined above is universal.

Further information concerning the universal bimorphisms is easily obtained by standard arguments using the Hahn-Banach Theorem: the image of $W$ in $F$ with respect to $h$ is actually $U \otimes V$, and for any $a \in|A|$ and $b \in|B|$ one has $\left\|h i_{a}(b)\right\|=\|a\|\|b\|=\left\|h j_{b}(a)\right\|$. This identifies the restriction of the norm of $F$ to $U \otimes V$ as the greatest cross norm; the Banach space $F$ is usually denoted $A \hat{\otimes} B$ and Proposition 3 now provides the natural equivalence $(A \hat{\otimes} B, C) \rightarrow$ $(A, H(B, C))$, given by $h \rightsquigarrow \tilde{h}$ where $\tilde{h}(a)(b)=h(a \otimes b)$.

An analogous, and in fact simpler, discussion applies to the subcategory $\mathrm{Ban}_{1}$ of Ban given by the linear contractions, i.e. the $f: A \rightarrow B$ in Ban such that $\mid f \| \leq 1$, as F. E. J. Linton has kindly pointed out to us. Here, the underlying set functor is the unit ball functor, and $H$ is taken as before. The relation $\|H(f, g)\| \leq\|f\|\|g\|$, noted above for Ban, then shows $H$ is also an internal 
hom-functor for $\mathrm{Ban}_{1}$. The functionality of $H$ for $\mathrm{Ban}_{1}$ is an immediate consequence of Proposition 1: $\mathrm{Ban}_{1}$ has concrete products, and restricting the $f \in H(A, B)$ to the unit ball $|A|$ of $A$ provides an embedding $H(A, B) \rightarrow B^{|A|}$ of the required kind. Finally, the existence of universal bimorphisms follows now from Proposition 4 since $\mathrm{Ban}_{1}$ has coproducts (Semadeni [15]) and, like Ban, satisfies the remaining hypotheses of that proposition. It should be noted that the resulting tensor multiplication in $\mathrm{Ban}_{1}$ is the same as in Ban.

(5) If $R$ is a commutative ring, then the (full subcategory $R \operatorname{Mod}_{f g p}$ of the category $R$ Mod of all $R$-modules given by the finitely generated projective $A \in R$ Mod is closed with respect to the usual internal hom-functor $H$ of $R$ Mod and has $R$, as module over itself, as dualizer. To see this, we recall that the $A \in R \operatorname{Mod}_{f g p}$ are exactly the modules isomorphic to direct summands of finite powers $R^{n}$ of $R$ (Lambek [7]). One readily obtains that $H(A, B)$ is isomorphic to a direct summand $H\left(R^{n}, R^{m}\right) \cong R^{n m}$ if $A$ and $B$ are isomorphic to direct summands of $R^{n}$ and $R^{m}$ respectively, which shows the asserted closure property. As to the dualizer condition, the natural homomorphism $A \rightarrow A^{* *}=H(H(A, R), R)$ is easily seen to be an isomorphism for $A=R^{n}$, and a simple argument shows that the direct summands of any $A$ with this property inherit this property. Since $H$ is clearly functional, it now follows that the functor $T$, given by $T(A, B)=H\left(A, B^{*}\right)^{*}$, is a tensor multiplication relative to $H$ on $R \operatorname{Mod}_{f g p}$. Moreover, one also has that this subcategory of $R$ Mod is closed with respect to the usual tensor product $\otimes$ resulting from the universal bilinear maps of $R$ Mod, and this establishes natural isomorphisms $A \otimes B \cong$ $\operatorname{Hom}\left(A, B^{*}\right)^{*}$ for all finitely generated projective modules $A, B \in R$ Mod. For the case of vector spaces, i.e. where $R$ is a field, this is a well-known fact, often utilized in text books to introduce the tensor product of finite dimensional vector spaces.

There is a topological variant of the above. If $R$ is now a topological commutative ring with unit, let $R$ Mod be the category of topological $R$ modules, in the sense that the action $R \times A \rightarrow A$ of $R$ on the topological group $A$ is continuous, with continuous module homomorphisms as maps. Then, again, one has an internal hom-functor $H$, where the module structure on the set of all $h: A \rightarrow B$ in $R$ Mod is the usual one and the topology is the topology of pointwise convergence. One readily sees that the usual action $R \times$ $H(A, B) \rightarrow H(A, B)$ is indeed continuous so that $H(A, B) \in R$ Mod; also, for any $f: C \rightarrow A$ and $g: B \rightarrow D$ the usual linear map $H(f, g)$ is continuous. Moreover, $H$ is functional, by Proposition 1 .

Now; for any $A, B \in R$ Mod, one can take $T(A, B)$ as the usual tensor product $A \otimes B$ of the underlying modules, with the weak topology relative to all linear maps

$$
A \otimes B \rightarrow C, \quad a \otimes b \leadsto h(a)(b)
$$


for $h: A \rightarrow H(B, C)$. Then $x \leadsto x \otimes b$ and $z \rightsquigarrow a \otimes z$ are continuous linear maps $A \rightarrow T(A, B)$ and $B \rightarrow T(A, B)$ for any $b \in B$ and $a \in A$. Moreover, the usual action $R \times T(A, B) \rightarrow T(A, B)$ is continuous, as one sees by following it by any linear map $A \otimes B \rightarrow C$ of the above form, and in the same way one checks that $T$ associates continuous linear maps with such maps. These facts then readily imply that $T$ is a tensor multiplication relative to $H$.

Further properties of $T$ and $H$ are, as in the non-topological case, that $H(A \oplus B, C) \cong H(A, C) \oplus H(B, C), H(A, B \oplus C) \cong H(A, B) \oplus H(A, C)$, and the analogous conditions for $T$, the isomorphisms being provided by the obvious maps and natural in $A, B$, and $C$. In addition, one has $H\left(R^{n}, R^{m}\right) \cong$ $R^{n m} \cong T\left(R^{n}, R^{m}\right)$.

Now let $K$ be the full subcategory of $R$ Mod given by the $A \in R$ Mod isomorphic to direct summands of some $R^{n}$, i.e. the $A \in R$ Mod which are finitely generated and projective with respect to onto maps (note that all linear maps $R^{n} \rightarrow R^{m}$ are continuous). Then, by the properties of $T$ and $H$ mentioned above, $K$ is closed under the action of $T$ and $H$, and this provides $K$ with an internal hom-functor and a tensor multiplication relative to it. Also, $R$ is again a dualizer, and hence one has natural isomorphisms $T(A, B) \cong$ $H\left(A, B^{*}\right)^{*}$, with the same effect as in the non-topological case. This can then be viewed as an alternative description of the topology of $T(A, B)$.

A further module category with a dualizer, also a generalization of finitedimensional vector spaces, is the category $R$ mod of finitely generated modules over a commutative artinian ring $R$ with unit. $R$ mod is closed, in $R$ Mod, with respect to the usual internal hom-functor, the tensor product, and the formation of injective hulls. In particular, the injective hull $I$ of $R$ modulo its radical is a dualizer (Morita [11]), a consequence of the fact that dualizing relative to $I$ preserves the length of composition series. Again, as before, one then has a natural isomorphism $A \otimes B \rightarrow \operatorname{Hom}\left(A, B^{*}\right)^{*}$. Incidentally, for suitable $R$ one has $I^{*} \not \equiv I$, unlike the case of finitely generated projective modules, or CJSL. A ring of this type is the polynomial ring $K[x, y]$ over a field modulo the ideal $\left(x^{2}, x y, y^{2}\right)$; here the socle of $R$ has length 2 but that of $I$ has length 1 so that $I \not R \cong I^{*}$.

An analogous situation, generalizing the case of finite-dimensional vector spaces in a different direction, is given by the finitely generated semi-simple (= completely reducible) modules over a commutative semi-local ring $R$ with unit, the dualizer being $R$ modulo its radical. Incidentally, for commutative $R$ with infinitely many maximal ideals, one still has a self-duality for the module category in question, given by dualizing with respect to the direct sum of all $R / M$ ( $M$ maximal ideal of $R$ ), even though this is not finitely generated. This self-duality is of the type discussed in the Remark following Lemma 1, but it is obviously not representable by a dualizer (within the category).

(6) Let $\mathbf{K}$ be the category of locally compact abelian groups isomorphic to a 
product of the form $F \times \mathbb{Z}^{n} \times \pi^{k}$, where $F$ is finite, $\mathbb{Z}$ the additive group of integers, and $T$ the circle group, the exponents being arbitrary natural numbers. If $H(A, B)$ is the group of continuous homomorphisms $A \rightarrow B$ with the compact-open topology then $H(A, B) \in \mathbf{K}$ for any $A, B \in \mathbf{K}$, as one sees by checking this for the special cases $A, B=$ finite, $\mathbb{Z}^{n}$, or $\pi^{k}$, for which the resulting $H(A, B)$ are again of that kind-a reduction which is justified since $H(A \oplus B, C) \cong H(A, C) \oplus H(B, C) \quad$ and $\quad H(A, B \oplus C) \simeq H(A, B) \oplus$ $H(A, C)$. Now, the maps $H(A, B) \rightarrow H(C, D)$ induced by any $f: C \rightarrow A$ and $g: B \rightarrow D$ are known to be continuous (Bourbaki [1], Ch. X, §3.4), and thus $H$ is an internal hom-functor for $\mathbf{K}$. Furthermore, $H$ is functional: any $H(A, B)$ is locally compact, and hence has an open cover on which the compact-open topology and the topology of pointwise convergence coincide, which means that these topologies in fact coincide on all of $H(A, B)$.

It now follows from Pontryagin Duality that $\pi$ is a dualizer for $\mathbf{K}$, and thus provides $\mathbf{K}$ with universal bimorphisms. In this case, $\mathbb{T}^{*}=\mathbb{Z}$ so that here one has another dualizer which is not isomorphic to itself.

(7) The non-additive counterpart to modules over commutative rings are $M$-sets where $M$ is a commutative monoid. Clearly, by the discussion in (1), every such category has a functional internal hom-functor and universal bimorphisms. If $M$ is actually a group, the $M$-set $U(A, B)$ can be described explicitly as follows: on the $M$-set $|A| \times B,|A|$ here the underlying set of $A$ with trivial action, the relation $\equiv$, defined such that $(a, b) \equiv(x, y)$ iff $x=s a$, $y=s^{-1} b$ for some $s \in M$, is a congruence and $U(A, B)=|A| \times B / \equiv$, the universal bimorphism being the quotient map modulo $\equiv$.

Certain counterparts to the facts concerning modules also hold in the present setting, at least under suitable assumptions. For instance, $M$, taken as $M$-set, is a dualizer for the finitely generated projective $M$-sets if $M$ is a group; however, this does not hold in general, the multiplicative monoid $\{0,1\}$ being a counterexample.

(8) An example analogous to (1) is obtained by considering, for a (possibly proper) class $\Gamma$ and $\sigma=\left(n_{\nu}\right)_{\nu \in \Gamma}$ any $\Gamma$-indexed family of cardinal numbers $\geq 1$, the category $\operatorname{Rel}(\sigma)$ of (purely) relational structures of type $\sigma$, whose objects are the relational structures $A=\left(X,\left(R_{\nu}\right)_{\nu \in \Gamma}\right)$ with underlying set $|A|=X$ and $\nu$ th relation $\nu_{A}=R_{\nu} \subseteq X^{n_{\nu}}$, its maps being the set maps which preserve all the relations, i.e. the set maps $h:|A| \rightarrow|B|$ with $h^{n_{\nu}}\left(\nu_{A}\right) \subseteq \nu_{B}$ are exactly the maps underlying homomorphisms in $\operatorname{Rel}(\sigma)$.

Any full subcategory $\mathbf{K}$ of $\operatorname{Rel}(\sigma)$ which is productive and hereditary (in the natural substructure sense) has a functional internal hom-functor $H: H(A, B)$ is the subobject of $B^{|A|}$ given by the $K$-maps $A \rightarrow B$, i.e. the $\nu$ th relation of $H(A, B)$ is the set of all $\left(f_{i}\right)_{i<n_{v}}$ such that $\left(f_{i}(x)\right)_{i<n_{v}} \in \nu_{B}$ for all $x \in A$. Moreover, $\mathbf{K}$ has coproducts by the construction due to Golema [5], and Proposition 4 is applicable; thus $\mathbf{K}$ has universal bimorphisms which provide a tensor multiplication $T$ for $H$. 
A more direct description of $T(A, B)$ is as follows: for each $\nu \in \Gamma$, take the $n_{\nu}$-ary relation

$$
R_{\nu}=\left\{((s(i), b))_{i<n_{\nu}}\left|s \in \nu_{A}, b \in\right| B \mid\right\} \cup\left\{((a, t(i)))_{i<n_{\nu}}|a \in| A \mid, t \in \nu_{B}\right\}
$$

on $|A| \times|B|$; then $T(A, B)$ is the $\mathbf{K}$-reflection of the resulting $\sigma$-relational structure.

If $\mathbf{K}$ is the category of partially ordered sets, one readily sees that $T(A, B)=$ $A \times B$, which provides a proof for the familiar fact that the category is cartesian closed.

Finally, we note that none of these categories can have a dualizer since || is represented by a one-point structure.

(9) We conclude with a somewhat curious example. For a monoid $M$ with unit $e$, the natural underlying set functor is the functor represented by $e$, i.e. $|e|=X$, the underlying set of $M$, and for each $s \in M,|s|$ is the action of $s$ on $X$ by left translation. Since | | associates with each pair of elements $s, t \in M$ the map $(s, t): x \leadsto t x s$, an internal hom-functor $H$ of $M$ must have the property that, for $u=H(s, t), u x=t x s$ for all $x \in M$. This implies that $u=t s$, and then $t s x=t x s$ for all $x \in M$, which makes $M$ commutative and $H$ the multiplication map; conversely, if $M$ is commutative then the multiplication map is indeed an internal hom-functor.

Let $M$ now be commutative. One then readily checks that (F1) holds, the single map whose existence is required being the unit $e$. Similarly, (F2) is satisfied, for if $f: X \rightarrow X$ is any map such that, for each $b \in M$, there exists a $w_{b} \in M$ for which $|f()|(b)=\left|w_{b}\right|$ it follows that $f(a)=f(a) e=|f(a)|(e)=$ $\left|w_{e}\right|(a)=w_{e} a$, and hence $f=\left|w_{e}\right|$. Hence the internal hom-functor is functional. Moreover, since $H(, e)$ is the identity automorphism, $e$ is a dualizer for $M$. Finally, the associated tensor multiplication $T$ is just the multiplication map of $M$, i.e. $T=H$.

\section{REFERENCES}

1. N. Bourbaki, General Topology II (Herrman, Paris and Addison-Wesley, Reading, Mass., 1966).

2. R. Brown, Function spaces and product topologies, Quart. J. of Math. 15 (1964) 238-250.

3. N. Dunford and J. T. Schwartz, Linear operators I. Interscience Publishers, New York 1958.

4. S. Fajtlowicz, Duality for algebras, (Unpublished manuscript).

5. K. Golema, Free products of compact general algebras. Coll. Math. 13 (1965) 165-166.

6. H. Herrlich, Regular categories and regular functors. Can. J. Math. 26 (1974), 709-720.

7. J. Lambek, Lectures on Rings and Modules, (Blaisdell, Waltham, Mass., 1966).

8. F. E. J. Linton, Autonomous equational categories, J. Math. Mech. 15 (1966) 637-642.

9. S. MacLane, Categories for the Working Mathematician, Springer, 1971.

10. E. Manes, Algebraic Theories, Graduate Texts in Mathematics 26, Springer-Verlag 1976.

11. K. Morita, Duality for modules and its applications to the theory of rings with minimum condition. Sci. Rep. Tokyo Kyoiku Daigaku 6 (1958), 83-142.

12. D. J. Mowatt, A Galois problem for mappings. Dissertation, University of Waterloo, 1968.

13. A. Pultr, Tensor products in the categories of graphs. Combinatorial Structures and their Applications. Proc. of the Calgary Conference, 1969, 327-330. Gordon and Breach 1970. 
14. D. Pumplün, Das Tensorprodukt als universelles Problem. Math. Ann. 171 (1967), 247-262.

15. Z. Semadeni, Banach Spaces of Continuous Functions, Volume I. Warsaw 1971.

16. H. H. Schaefer, Topological vector spaces. GTM3, Springer Verlag, New York-HeidelbergBerlin, 1971.

17. Z. Shmuely, Structure of Galois connections. Pac. J. 54 (1974), 209-225.

18. A. Waterman, Colloquium lecture at McMaster University, October 1966.

Department of Mathematics

MCMASTER UNIVERSITY

1280 MAIN STREET

Hamilton, Ontario

CANADA L85 4K1 